\title{
Thermal, mechanical, and physical properties of seaweed/sugar palm fibre reinforced thermoplastic sugar palm Starch/Agar hybrid composites
}

\begin{abstract}
The aim of this research is to investigate the effect of sugar palm fibre (SPF) on the mechanical, thermal and physical properties of seaweed/thermoplastic sugar palm starch agar (TPSA) composites. Hybridized seaweed/SPF filler at weight ratio of 25:75, 50:50 and 75:25 were prepared using TPSA as a matrix. Mechanical, thermal and physical properties of hybrid composites were carried out. Obtained results indicated that hybrid composites display improved tensile and flexural properties accompanied with lower impact resistance. The highest tensile (17.74 $\mathrm{MPa}$ ) and flexural strength (31.24 MPa) was obtained from hybrid composite with 50:50 ratio of seaweed/SPF. Good fibre-matrix bonding was evident in the scanning electron microscopy (SEM) micrograph of the hybrid composites' tensile fracture. Fourier transform infrared spectroscopy (FT-IR) analysis showed increase in intermolecular hydrogen bonding following the addition of SPF. Thermal stability of hybrid composites was enhanced, indicated by a higher onset degradation temperature $\left(259{ }^{\circ} \mathrm{C}\right)$ for 25:75 seaweed/SPF composites than the individual seaweed composites $\left(253{ }^{\circ} \mathrm{C}\right)$. Water absorption, thickness swelling, water solubility, and soil burial tests showed higher water and biodegradation resistance of the hybrid composites. Overall, the hybridization of SPF with seaweed/TPSA composites enhances the properties of the biocomposites for short-life application; that is, disposable tray, plate, etc.
\end{abstract}

Keyword: Thermoplastic starch; Sugar palm composites; Hybrid composites 